\title{
Exposure to Discretionary Arrests Increases Support for Anti-Police Protests ${ }^{*}$
}

\author{
Ted Hsuan Yun Chen ${ }^{\text {ab }} \quad$ Paul McLachlan ${ }^{c} \quad$ Christopher J. Fariss $^{\mathrm{d}}$
}

September 28, 2021

\begin{abstract}
The legitimacy of the state rests on individuals' perceptions of fairness when interacting with state institutions and state agents. The police as an institution and as individual agents have wide latitude to detain and use force against individuals. We argue that encounters with state bureaucracy and civil servants, specifically the police, can generate individual-level grievances against the state, and that these grievances make it more likely an individual participates in protest against the state. We study support for and the legitimacy of policing in the context of the anti-police protests in Baltimore, MD following the death of Freddie Gray in April, 2015. Using data from police records and social media, we show that individuals with higher exposure to discretionary arrests - arrests that are potentially viewed as illegitimate or arbitrary — are more likely to support protests against the police. In contrast, we demonstrate that exposure to arrests for major crimes such as murder does not follow the same pattern. Thus, support for the police as an institution varies systematically with exposure to arbitrary and capricious encounters with police agents. As these grievance generating encounters become more widespread, we expect to see increased protests against the police and further erosion in support of the police as an institution. Alternatively, shifting institutional resources to focus on major crimes and limiting the discretionary authority of police agents when interacting with the public may help to repair the legitimacy of policing institutions over the long term.
\end{abstract}

Keywords: discretionary arrests; protest participation; social media data

\footnotetext{
${ }^{*}$ Corresponding author: Christopher J. Fariss (cjfariss@umich.edu). J.Fowler, C.Liu, K.Reuning, Z.Steinert-Threlkeld; participants at CSSSA'17, Midwest Political Science Association, PolMeth, and PolNet; and workshop participants at Emory, Georgia State University, University of Georgia, University of Houston, and University of Washington provided helpful feedback. Paul Rodriguez provided invaluable help with scientific computing. We acknowledge research support from the Charles Koch Foundation, Emory's Institute for Quantitative Theory and Methods, the National Science Foundation's XSEDE program (SES170013), and the San Diego Supercomputer Center.

${ }^{a}$ Faculty of Social Sciences, University of Helsinki

${ }^{\mathrm{b}}$ Department of Computer Science, Aalto University

${ }^{\mathrm{c} S i m B i o S y s}$ Lab, Department of Computer Science, Emory University

${ }^{\mathrm{d}}$ Department of Poltical Science, University of Michigan
} 


\section{Contents}

1 Introduction 1

2 Materials and Methods 4

2.1 Protest Support . . . . . . . . . . . . . . . . . . . . . 4

2.2 Arresting Visits . . . . . . . . . . . . . . . . . . . . 5

2.3 Individual Geoprofiles . . . . . . . . . . . . . . . . . 6

2.4 Additional Results . . . . . . . . . . . . . . . . . . . 7

$\begin{array}{lr}\text { References } & 8\end{array}$

$\begin{array}{lr}\text { A Protest Engagement Classifier } & 9\end{array}$

$\begin{array}{lr}\text { B Geoprofile Inference } & 10\end{array}$ 


\section{Introduction}

Baltimore Police Department (BPD) officers arrested Freddie Gray on April 12, 2015 for suspected possession of an "illegal switchblade." Gray fell into a coma for reasons that are disputed while being transported in a police van. He did not recover from his injuries and died on April 19. To denounce Gray's alleged mistreatment and BPD brutality, protesters began gathering in front of the Western District Police Station on April 18. These protests grew rapidly in size throughout the week and eventually reached several thousand people and only ended after the Maryland National Guard imposed a mandatory curfew from April 28 to May 3. These protests, collectively referred to as the Freddie Gray protests, are our empirical focus. Who joined these protests and who supported them?

We argue that encounters with state bureaucracy and civil servants can generate individuallevel grievances, and that these grievances make it more likely an individual participates in protests events. This is because civil servants, such as teachers, social workers, and law enforcement agents, provide important information about an individual's position in society. These civil servants or "street-level bureaucrats" wield enormous power over citizens' lives Lipsky (2010). They determine whether a permit is issued; a child is removed from their parent's custody; a fine is imposed; or an arrest or police visit is made. Thus, in the eyes of an individual, every interaction with a street-level bureaucrat either reaffirms the legitimacy of the state and its agents or generates a new grievance Lipsky (2010); White, Nathan and Faller (2015). As the level of illegitimate or arbitrary interactions with street-level bureaucrats increases, the intensity of an individual's grievances also increases.

Though our argument about the origin of grievances against the state generalizes to interactions with all bureaucrats, our empirical focus in this paper is the effect of encounters with police officers on grievances and protest mobilization. Interacting with police officers is rarely pleasant and people of color must be especially vigilant to protect their physical integrity Davenport, Soule and Armstrong (2011); Pierson et al. (2020). This observation is highlighted by the growing literature on the consequences of interactions with the "carceral state," which shows that police-citizen interactions decrease trust in government and participation in politics through formal institutions Kang and Dawes (2017); Tyler, Fagan and Geller (2014); Lerman and Weaver (2014); Pierson et al. (2020); Streeter (2019); Wildeman (2014); Williamson, Trump and Einstein (2018); Uggen and Manza (2002); Weaver, Prowse and Piston (2019). Moreover, these negative effects also propagate locally to those who did not interact directly with the police Burch (2013); Williamson, Trump and Einstein (2018). If the level of illegitimate or arbitrary interactions with police officers increases, then the intensity of an individual's grievances against the police also increases Weaver, Prowse and 
Piston (2019). We hypothesize that an individual with pre-existing grievances against the police is more likely to protest against the police during periods of mobilization such as the Freddie Gray protests than an individual without such grievances.

\section{Results}

Using data from police records and social media, with a specific focus on costly communication online Earl et al. (2015), we show that individuals with grievances because of higher exposure to discretionary arrests in their communities - arrests that are potentially viewed as illegitimate or arbitrary such as those for possession of marijuana and other drugs - are more likely to support protests against the police. In contrast, we demonstrate that exposure to arrests for major crimes such as murder, or those that have the potential for a maximum of a life sentence, do not follow the same pattern.

To control for characteristics of each user's communities, we access information about the racial and demographic makeup of each Baltimore census tract as provided by the U.S. Census Bureau. Specifically, we include in our model the proportion of the population that is (1) Black, (2) Hispanic, (3) female, (4) between the ages of 15 and 24 (i.e. the youth bulge), (5) at least a high school graduate; (5) the median income; the (6) poverty and the (7) unemployment rates; and (8) the overall population.

The main independent variable is one of four types of exposure to policing as described above: Arresting Visits for the (1) crime of murder, (2) any crime with a maximum of life in prison, (3) drug possession, and (4) marijuana possession. All other variables that enter the four models are the same. The four generalized linear regression $(\mathrm{glm})$ models with a logit link function for the binary dependent variable Protest Support is as follows:

Results from our models are presented in Figure 1, which shows a strong positive association between Protest Support and membership in a community that experiences discretionary arrests such as arrests for drugs and marijuana (i.e., grievances generated because of an experience with Arresting Visits). In contrast, protest participation is not associated with membership in a community that experiences arrests for murder and arrests with a maximum charge of life in prison.

\section{Discussion}

People who tweeted during the protests used many pro-Freddie Gray and anti-police hashtags. These individuals were also more likely to join the protests at one of the many government buildings at which the Freddie Gray protests took place in Baltimore in April 2015. 


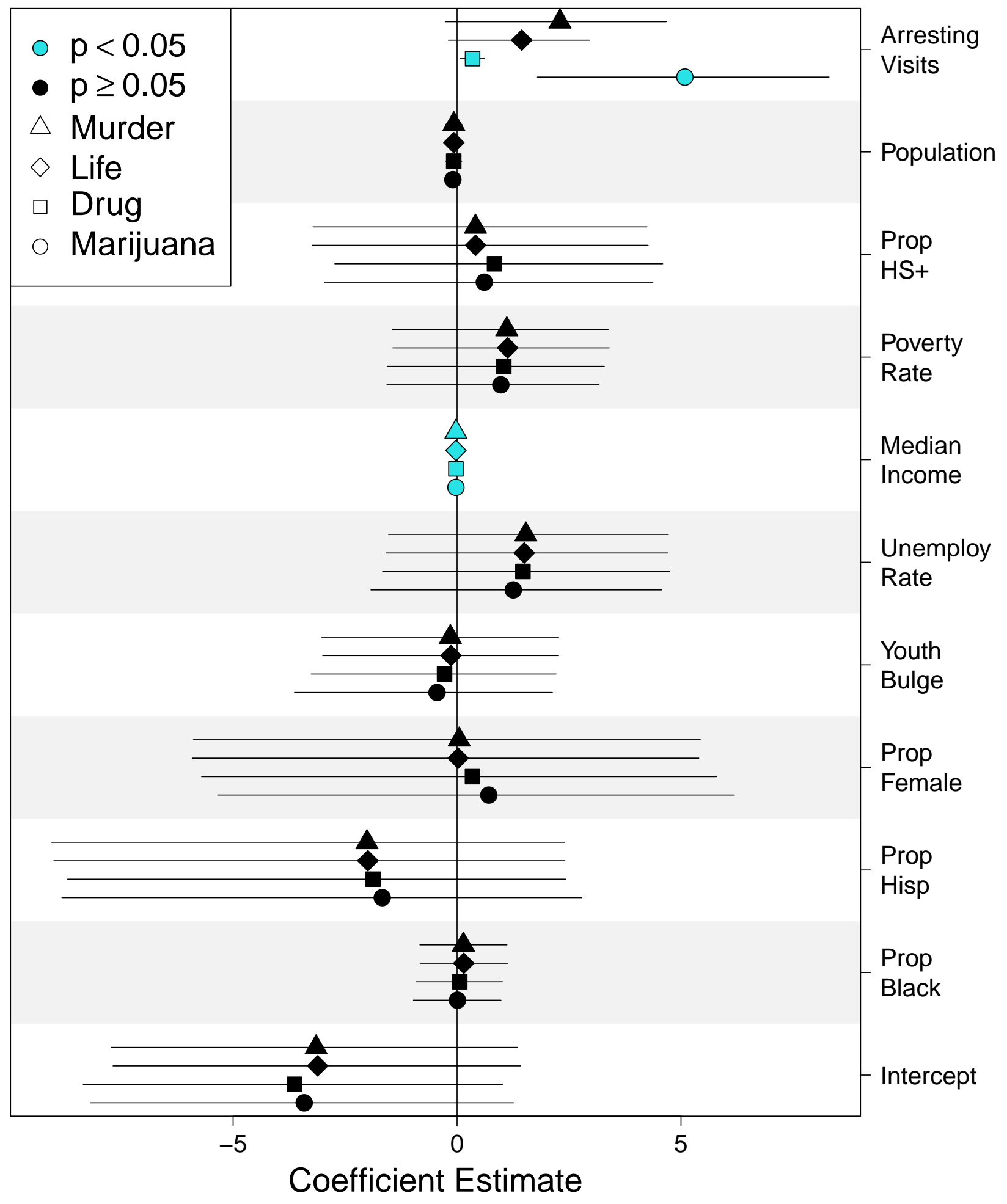

Figure 1: Coefficient estimates for models of protest support. Statistical significance and $95 \%$ confidence intervals are based on 2,000 bootstrapped samples, and also incorporates uncertainty in our estimation of the users' communities. 
These individuals also reside in the areas of Baltimore that are over-policed and more likely to experience the types of arbitrary and capricious interactions with state agents that generate grievances Weaver, Prowse and Piston (2019).

Thus support for the police during a period of protest varies systematically with exposure to arbitrary and capricious encounters with police agents. If these grievance generating encounters become more widespread, we expect to see more individuals willing to criticise the police and join protest once they begin, which may lead to increased protests against the police and possibly further erosion in support of the police as an institution. Alternatively, shifting institutional resources to focus on major crimes and limiting the discretionary authority of police agents when interacting with the public may help to repair the legitimacy of policing institutions over the long term.

Our sample is a set of 2,232 Twitter users who made at least one geotagged tweet from within Baltimore, MD during the Freddie Gray protests (April 16 to May 4, 2015) and whose geoprofiles we estimated to fall in Baltimore City bounds (see SI Appendix). The empirical relationship between Protest Support and Arresting Visits that we discussed above is based on our measurement strategy that uses the content of the tweets to operationalize the dependent variable Protest Support and the geographic proximity of the Twitter user to police activity that we use to operationalize the independent variables Arresting Visits.

\section{Materials and Methods}

\section{$2.1 \quad$ Protest Support}

For each Twitter user, we infer protest support by looking at their expressions of anti-police sentiment on Twitter, including explicit support for the Freddie Gray protests.

We began by obtaining all tweets geotagged to be inside Baltimore City bounds during the Freddie Gray protests. This sample included 111,440 tweets from 7,884 accounts, and was licensed for use from Twitter. We arrived at our measure of protest support in two steps. First, we inferred whether a tweet was engaging with the protests. We randomly sampled

baltimoreuprising, justiceforfreddiegray, blacklivesmatter, march2justice, nojusticenopeace, endthecurfew, phillytobaltimore, handsupdontshoot, 300menmarch, ferguson, fuckthepolice, accountability, blackpower, nojustice, policebrutality, trayvonmartin, athleteally, civilprotests, policestate, wewantjustice, blacklawyersforjustice, endpolicebrutality, mikebrown

Table 1: Protest support hashtags with more than 2 occurrences. Variants were grouped together (e.g. BLM and BlackLivesMatter). 
5,000 tweets and manually labelled them as protest-engaging or not. When labelling for protest engagement, we do not differentiate between positive (e.g. anti-police), negative (e.g. criticizing riots), and neutral (e.g. news) stances. Using $80 \%$ of this hand-labelled data, we trained a machine learning classifier (soft vote of a regularized logistic regression and a support vector classifier with feature selection). Based on cross-validation using the remaining $20 \%$ test data, our model has a $95.12 \%$ accuracy and $13.92 \%$ false positive rate. Details of our machine learning classifier are presented in the SI Appendix.

Second, from the set of protest engagement tweets, we further subset to protest support tweets. From the set of engagement tweets, we extracted all hashtags (totalling 1,417) and manually labelled ones that are explicitly indicative of (1) participation in the Freddie Gray protests or support for it, (2) anti-police sentiment, or (3) anti-racism or black empowerment sentiment. In many cases, these hashtags were attached to tweets that clearly indicate the account to be physically present at a protest event happening in Baltimore City. We took a conservative approach, meaning that hashtags used for generic solidarity (e.g. \#baltimore) or mourning (e.g. \#ripfreddiegray) were not included. Then, we classified all tweets containing at least one of these 96 hashtags, see the most frequent examples in Table 1, to be protest support tweets. Aggregating to the account-level by whether the account used at least one of these hashtags is a supporter, we get a total of 338 supporters out of 7,884 users.

\subsection{Arresting Visits}

To measure each user's prior experience with policing, we first computed census tract-level policing using data obtained from a public records request to the Baltimore Police Department (BPD), then estimated each user's exposure to these incidences based on their inferred 'geoprofile' following the procedure described in Chen, Zachary and Fariss (2017). The BPD data contains $\log$ for all felony arrests from the years 2012-2015, which include the date the arrest was made; the location where the arrest was made; the charges; and basic demographic information about the arrested (i.e. age, sex, and race). In total, the data used in our analysis contains 15,587 felony arrests made by the BPD from January 1, 2012 to April 17, 2015, one day before the start of the Freddie Gray protests.

To place these arrests in census tracts, we first converted the street address provided with each arrest to geographical coordinates using Google's geocoding application programming interface (API). Because we intend to capture neighborhood-level exposure, highway arrests are removed. Additionally, we made a small number of manual adjustments when the API returned no match due to spelling errors or unavailable street numbers. Next, we converted the geographical coordinates to census tracts using the Federal Communications 


\section{Commission's Block API.}

To examine whether grievances increase with perceived unfair treatment, we subset our data according to whether the arresting officer has discretion in making the arrest. To do so, we used the initial charge recorded in the arrest logs. While the specifics of the charging document might change between the time individuals are first arraigned and when they are brought to trial, it is a useful proxy of the type of activity the arrested individual was engaged in. For each arrest, we consulted the 2017 Maryland Sentencing Guidelines Offense Table to code all associated charges according to their offense type and maximum term. Using this data, we labelled arrests related to a) marijuana and b) other drugs as discretionary because only a small portion of drug users and sellers are ever arrested. In contrast, more severe crimes, which we capture using c) murders and d) all crimes with life sentences, should not induce grievances as police have lower discretion in making arrests in these scenarios. Finally, for each of the four arrest categories, we constructed a measure of exposure to policing, Visits Leading to Arrests, which treats all arrests with the same address on the same day as a single incident within the census tract.

\subsection{Individual Geoprofiles}

We measure individuals' exposure to different types of policing by extracting geographical information from their tweets to estimate their 'geoprofile', which is the set of locations the individual is consistently linked to (e.g. their home or workplace). Specifically, for each Twitter account in our sample, we obtained their 3,200 most recent tweets using Twitter's timeline API, and extracted all exact geographical coordinates contained in their geotagged tweets. These tweets were collected between July 19, 2016 and August 27, 2016. Because exact coordinates can only be tagged using a GPS, we can be confident individuals actually visited these locations, subject to inaccurate GPS measurements and the unlikely case of GPS spoofing.

After collecting this geolocation data, we followed the spatial clustering procedure outlined in Chen, Zachary and Fariss (2017) and inferred for each account a set of source locations from which its observed geotags are likely to have originated. The source locations collectively form that account's geoprofile. These geoprofiles allow us to estimate users'

exposure to policing based on where they physically spend time. Specifically, we measure grievances with georeferenced arrest data we obtained from from the BPD through a public records request. Details of our implementation are presented in the SI Appendix. 

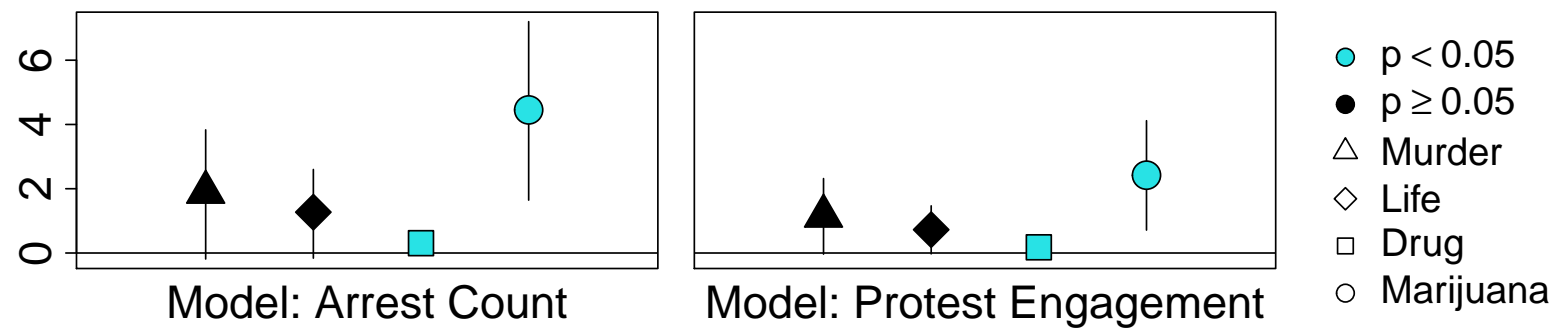

Figure 2: Coefficient estimates for the exposure to policing term on two alternative sets of models. The 'Arrest Count' model changes the exposure to policing from Arresting Visits to Arrest Counts; the 'Protest Engagement' model changes the outcome variable from Protest Support to Protest Engagement. Statistical significance and $95 \%$ confidence intervals are based on 2,000 bootstrapped samples, and also incorporates uncertainty in our estimation of the users' communities.

\subsection{Additional Results}

The results reported in Figure 1 are robust to a number of alternative specifications on our measures. First, we tested an alternative specification of the exposure to policing term, replacing the original count of Arresting Visits with a raw Arrest Count in the census tract. Second, we tested an alternative specification of protest support by removing the requirement of using a tweet containing one of the 96 hashtags (i.e. the second step of our original support classification scheme), which essentially means we use the Protest Engagement label from our first stage machine learning classifier. This is a more permissive classification, returning 2,124 individuals engaging with the protest.

The coefficients for the exposure to policing terms for all four types of arrests, for both alternative specifications, are presented in Figure 2. The models are fitted with the same control variables as the main model, which are not reported here. These models yield the same pattern as our main results. Individuals with higher exposure to discretionary arrests are more likely to support the anti-police protests while exposure to arrests for major crimes is not related to protest behavior. 


\section{References}

Burch, Traci. 2013. Trading democracy for justice: Criminal convictions and the decline of neighborhood political participation. University of Chicago press.

Chen, Ted Hsuan Yun, Paul Zachary and Christopher J Fariss. 2017. Social Activity Hubs: Estimating User Specific Contextual Factors from Social Media Data. In Proceedings of the 2017 International Conference of The Computational Social Science Society of the Americas. pp. 1-9.

Davenport, Christian, Sarah A Soule and David A Armstrong. 2011. "Protesting while black? The differential policing of American activism, 1960 to 1990." American Sociological Review 76(1):152-178.

Earl, Jennifer, Jayson Hunt, R Kelly Garrett and Aysenur Dal. 2015. New technologies and social movements. In The Oxford handbook of social movements. Oxford University Press Oxford pp. 355-366.

Hauge, Michelle V, Mark D Stevenson, D Kim Rossmo and Steven C Le Comber. 2016. "Tagging Banksy: Using geographic profiling to investigate a modern art mystery." Journal of Spatial Science 61(1):185-190.

Kang, Woo Chang and Christopher Dawes. 2017. "The Electoral Effect of Stop-and-Frisk." Available at SSRN 3000561 .

Lerman, Amy E and Vesla M Weaver. 2014. Arresting citizenship: The democratic consequences of American crime control. University of Chicago Press.

Lipsky, Michael. 2010. Street-level bureaucracy: Dilemmas of the individual in public service. Russell Sage Foundation.

Pierson, Emma, Camelia Simoiu, Jan Overgoor, Sam Corbett-Davies, Daniel Jenson, Amy Shoemaker, Vignesh Ramachandran, Phoebe Barghouty, Cheryl Phillips, Ravi Shroff et al. 2020. "A large-scale analysis of racial disparities in police stops across the United States." Nature human behaviour 4(7):736-745.

Rossmo, D Kim. 1999. Geographic profiling. CRC press.

Streeter, Shea. 2019. "Lethal force in black and white: Assessing racial disparities in the circumstances of police killings." The Journal of Politics 81(3):1124-1132.

Tyler, Tom R, Jeffrey Fagan and Amanda Geller. 2014. "Street stops and police legitimacy: Teachable moments in young urban men's legal socialization." Journal of empirical legal studies 11(4):751-785.

Uggen, Christopher and Jeff Manza. 2002. "Democratic contraction? Political consequences of felon disenfranchisement in the United States." American Sociological Review 67(6):777-803.

Verity, Robert, Mark D Stevenson, D Kim Rossmo, Richard A Nichols and Steven C Le Comber. 2014. "Spatial targeting of infectious disease control: identifying multiple, unknown sources." Methods in Ecology and Evolution 5(7):647-655.

Weaver, Vesla, Gwen Prowse and Spencer Piston. 2019. "Too much knowledge, too little power: An assessment of political knowledge in highly policed communities." The Journal of Politics 81(3):1153-1166.

White, Ariel R, Noah L Nathan and Julie K Faller. 2015. "What do I need to vote? Bureaucratic discretion and discrimination by local election officials." American Political Science Review 109(1):129-142.

Wildeman, Christopher. 2014. "Parental incarceration, child homelessness, and the invisible consequences of mass imprisonment." The ANNALS of the American Academy of Political and Social Science 651(1):7496.

Williamson, Vanessa, Kris-Stella Trump and Katherine Levine Einstein. 2018. "Black lives matter: Evidence that police-caused deaths predict protest activity." Perspectives on Politics 16(2):400-415. 


\section{A Protest Engagement Classifier}

To develop a classifier model of protest engagement tweets, we manually labelled a random sample of 5,000 tweets drawn from our full corpus of 111,400 tweets. We assigned tweets a 1 when its content indicates the user was engaging with the protest. When labelling for protest engagement, we do not differentiate between positive (e.g. anti-police), negative (e.g. criticizing riots), and neutral (e.g. news) stances. All other tweets were assigned 0. In this procedure, we classified 624 tweets (approximately 12\%) of our training set as protest engagement tweets.

Before fitting the classifier models, we preprocessed our data in two ways that mitigate difficulties associated with the sparsity and high dimensionality of the tweet text data. First, we relied on the tendency for hashtags to be written in camel case to split them into separate words whenever possible. The string "\#BlackLivesMatter", for example, becomes "Black Lives Matter." Second, we stemmed and normalized our corpus with NLTK's TweetTokenizer package. By stemming our corpus, we reduce inflected words to their word stem. To normalize our corpus and reduce dimensionality, we converted emojis and accented letters into unicode. After preprocessing, we randomly divide our manually coded tweets into a training (80\%) and a test (20\%) set for cross-validation.

We obtained feature vectors from our preprocessed data using term-frequency inversedocument frequency (TF-IDF). While other tokenization algorithms weigh every feature's importance by counting the number of times it appears in a corpus, TF-IDF improves upon this approach accounting for both the number of times each feature appears and the total number of words in the corpus. We allow our n-gram size to range from 1 to 5 and converted to binary with a threshold of 0 . We then convert these features into a vector in which each element represents a single feature (obtained via TF-IDF) and its value is its weight. In other words, we transform every tweet into a vector in which each unique word is an element and that element's value is the frequency that word appears in our corpus divided by the weight.

We fit two models on our training data, a regularized logistic regression and a support vector classifier with feature selection. As our logistic regression and support vector classifier performed similarly (F1 scores of 0.79 and 0.77 respectively), we averaged their predictions for our remaining corpus using soft voting, which averages the predicted probabilities across an ensemble of classifiers. The soft vote model performed better than both constituent models, with an F1 score of 0.81 . After cross-validation on our test set, we estimate that the final accuracy of our soft vote model to be $95.12 \%(\sigma=0.50)$ with a false positive rate of $13.92 \%$ $(\sigma=3.53)$. 


\section{B Geoprofile Inference}

To infer an individual's exposure levels to different types of policing, we estimated their geoprofile, which is the set of locations the individual is consistently linked to (e.g. their home or workplace) using volunteered geographical information embedded in their tweets. For each of the 7,884 Twitter accounts in our sample, we used Twitter's API endpoint (specifically the GET statuses/user_timeline from the v1.1 endpoint) to obtain their 3,200 most recent tweets (as of the data collection period between July 19 and August 27, 2016). We then extracted all exact geocoordinates contained in their geotagged Tweets. Because tweets can only be tagged with exact coordinates using a device's GPS, we can be confident individuals actually visited these locations, subject to inaccurate GPS measurements and GPS spoofing. Following concepts from spatial epidemiology Verity et al. (2014) and criminology Rossmo (1999); Hauge et al. (2016), we treat each geotagged tweet as an observed incident of the user's movement patterns from which we can infer their source locations, which collectively make up the user's geoprofile.

From the set of all observed incidents, we estimate the users' geoprofiles following the implementation, described in Chen, Zachary and Fariss (2017), of a Dirichlet process mixture (DPM) model for spatial data (Verity et al. 2014). This model is a mixture model of movement patterns in geographical space (i.e. latitude and longitude) where observed geotags are assumed to be drawn from an unfixed set of identical distributions centered on an equal number of source locations. Given the input set of observed geotags, the model returns clustered observations and their most likely source locations. One key advantage of the DPM model is that is does not require prior assumptions about the true number of source locations, but instead infers it from the data. Where there are multiple source locations, especially when they are geographically distant, a misspecified number will result in inaccurate source location estimates that are skewed by "outliers" that are actually observations originating from a different source.

In our implementation we assumed movement patterns to follow a bivariate normal distribution centered on source locations, with standard deviation of approximately 5 kilometers (0.05 degree decimals in Baltimore City). Analytical solutions are generally intractable for data with larger than 10 observed geotags Verity et al. (2014), so estimation was done using MCMC. For each account, we used 5 chains and allowed a maximum of 8,000 iterations for

model convergence. From our 7,884 users, we obtained 5,946 with geotagged tweets in the period studied, and of those, 5,419 had converged models. Based on each set of estimated source locations, we defined a user's geoprofile as the set of geotagged locations closest to each inferred source location. 
Using each user's geoprofile, we placed them in corresponding census tracts. Not all estimated source locations fall within Baltimore City bounds, but because we only have arrest data from the BPD, we dropped all source locations outside of Baltimore City. For users with more than one estimated source location within Baltimore City, we take the average statistics from all of their census tract. Note that given the parameters of the DPM model (i.e. the standard deviation of the bivariate normal distribution), it is rare for users to have more than two estimated locations within Baltimore City.

Finally, to account for uncertainty in the geoprofile estimation, all post-convergence steps were repeated with 100 different draws from the posterior distribution, and this uncertainty was incorporated into our logistic regression models. Depending on variations in these draws, our final sample ranges between 2,194 and 2,264. 